Supporting Information for:

\title{
Unusual Reactivity of Tris(pyrazolyl)borate Zirconium Benzyl Complexes
}

\author{
Han Lee and Richard F. Jordan* \\ Department of Chemistry, The University of Chicago \\ 5735 South Ellis Avenue, Chicago, Illinois 60637 \\ E-mail: rfjordan@uchicago.edu
}

\section{Contents}

I. Syntheses and Characterization of New Compounds

II. X-Ray Crystallography S18

$\begin{array}{lll}\text { III. ORTEP Diagrams } & \text { S23 }\end{array}$ 


\section{Synthesis and Characterization of New Compounds}

General procedures. All reactions were performed under purified $\mathrm{N}_{2}$ or vacuum using standard vacuum line techniques or in a $\mathrm{N}_{2}$-filled drybox. Nitrogen was purified by passage through columns containing activated molecular sieves and Q-5 oxygen scavenger. Pentane, hexanes, and toluene were distilled from sodium/benzophenone or purified by passage through columns of activated alumina and BASF R3-11 oxygen removal catalyst. Dichloromethane and dichloromethane- $d_{2}$ were dried over $\mathrm{CaH}_{2}$ and distilled. $\left[\mathrm{Ph}_{3} \mathrm{C}\right]\left[\mathrm{B}\left(\mathrm{C}_{6} \mathrm{~F}_{5}\right)_{4}\right]$ was obtained from Boulder Scientific and used as received. Benzyltributylammonium chloride was purchased from Aldrich and used as recieved. 2Butyne and $\mathrm{PMe}_{3}$ were purchased from Aldrich and dried over $4 \AA$ molecular sieves. Tetrabenzylzirconium $\left(\mathrm{Zr}\left(\mathrm{CH}_{2} \mathrm{Ph}\right)_{4}\right)$ and $\mathrm{KTp}^{*}\left(\mathrm{Tp}^{*}=\mathrm{HB}(3,5 \text {-dimethylpyrazolyl })_{3}\right)$ were prepared by literature procedures. ${ }^{1,2}$ Elemental analyses were performed by Midwest Microlabs.

NMR spectra were recorded at ambient temperature unless specified otherwise. ${ }^{1} \mathrm{H}$ and ${ }^{13} \mathrm{C}$ chemical shifts are reported relative to $\mathrm{SiMe}_{4}$ and were determined by reference to the residual ${ }^{1} \mathrm{H}$ and ${ }^{13} \mathrm{C}$ solvent resonances. ${ }^{11} \mathrm{~B}$ chemical shifts are reported relative to external $\mathrm{BF}_{3} \cdot \mathrm{Et}_{2} \mathrm{O},{ }^{19} \mathrm{~F}$ chemical shifts are reported relative to external $\mathrm{CFCl}_{3}$ and ${ }^{31} \mathrm{P}$ chemical are reported relative to external $85 \% \mathrm{H}_{3} \mathrm{PO}_{4}$. Coupling constants are given in Hz. ${ }^{1} J_{\mathrm{CH}}$ values were obtained from gated- $\left\{{ }^{1} \mathrm{H}\right\}{ }^{13} \mathrm{C}$ NMR spectra. NMR spectra of $\mathrm{B}\left(\mathrm{C}_{6} \mathrm{~F}_{5}\right)_{4}{ }^{-}$salts contain resonances at the free $\mathrm{B}\left(\mathrm{C}_{6} \mathrm{~F}_{5}\right)_{4}^{-}$anion positions. ${ }^{13} \mathrm{C}\left\{{ }^{1} \mathrm{H}\right\}$ NMR $\left(\mathrm{CD}_{2} \mathrm{Cl}_{2}\right): \delta 148.5(\mathrm{~d}, J=234), 138.6(\mathrm{~d}, J=246), 136.6(\mathrm{~d}, J=243)$,

\footnotetext{
${ }^{1}$ Zucchini, U., Albizzati, E., Giannini, U. J. Organometal. Chem. 1971, 26, 357.

2 Trofimenko, S. J. Am. Chem. Soc. 1967, 89, 6288.
} 
123.6 (br, ipso-C). ${ }^{19} \mathrm{~F}$ NMR $\left(\mathrm{CD}_{2} \mathrm{Cl}_{2}\right): \delta-133.2(\mathrm{br} \mathrm{s}, 2 \mathrm{~F}, o-\mathrm{F}),-163.7(\mathrm{t}, J=23,1 \mathrm{~F}, p-$ F), $-167.6(\mathrm{t}, J=19,2 \mathrm{~F}, m-\mathrm{F}) .{ }^{13} \mathrm{C}\left\{{ }^{1} \mathrm{H}\right\} \operatorname{NMR}\left(\mathrm{CD}_{2} \mathrm{Cl}_{2},-60{ }^{\circ} \mathrm{C}\right): \delta 147.5(\mathrm{~d}, J=241)$, $137.8(\mathrm{~d}, J=238), 135.8(\mathrm{~d}, J=249), 123.6(\mathrm{br}$, ipso-C $) .{ }^{19} \mathrm{~F} \mathrm{NMR}\left(\mathrm{CD}_{2} \mathrm{Cl}_{2},-60{ }^{\circ} \mathrm{C}\right): \delta-$ 133.7 (br s, 2F, o-F), -163.0 (t, $J=23,1 \mathrm{~F}, p-\mathrm{F}),-167.0(\mathrm{t}, J=19,2 \mathrm{~F}, m-\mathrm{F}) . \quad$ NMR spectra of cationic compounds generated in situ contain resonances for $\mathrm{Ph}_{3} \mathrm{CCH}_{2} \mathrm{Ph} .{ }^{1} \mathrm{H}$ $\operatorname{NMR}\left(\mathrm{CD}_{2} \mathrm{Cl}_{2}\right): \delta 7.29-7.12\left(\mathrm{~m}, 15 \mathrm{H}, \mathrm{CPh}_{3}\right), 7.04\left(\mathrm{t}, J=7.4,1 \mathrm{H}, p-\mathrm{CH}_{2} \mathrm{Ph}\right), 6.96(\mathrm{t}, J=$ 7.6, $\left.2 \mathrm{H}, m-\mathrm{CH}_{2} \mathrm{Ph}\right), 6.63\left(\mathrm{~d}, J=7.4,2 \mathrm{H}, o-\mathrm{CH}_{2} \mathrm{Ph}\right), 3.95\left(\mathrm{~s}, 1 \mathrm{H}, \mathrm{CH}_{2}\right) .{ }^{1} \mathrm{H}$ NMR $\left(\mathrm{CD}_{2} \mathrm{Cl}_{2},-60^{\circ} \mathrm{C}\right): \delta 7.23-7.15\left(\mathrm{~m}, 15 \mathrm{H}, \mathrm{CPh}_{3}\right), 7.01\left(\mathrm{t}, J=7.3,1 \mathrm{H}, p-\mathrm{CH}_{2} \mathrm{Ph}\right), 6.92(\mathrm{t}, J=$ 7.6, $\left.2 \mathrm{H}, m-\mathrm{CH}_{2} \mathrm{Ph}\right), 6.53\left(\mathrm{~d}, J=7.6,2 \mathrm{H}, o-\mathrm{CH}_{2} \mathrm{Ph}\right), 3.89\left(\mathrm{~s}, 2 \mathrm{H}, \mathrm{CH}_{2}\right) .{ }^{13} \mathrm{C}\left\{{ }^{1} \mathrm{H}\right\}\left(\mathrm{CD}_{2} \mathrm{Cl}_{2}\right.$, $\left.-60{ }^{\circ} \mathrm{C}\right): \delta 146.2,138.0,130.8,129.3,127.4,126.9,125.7,125.7,58.2\left(\mathrm{Ph}_{3} C\right), 44.8$ $\left(\mathrm{CH}_{2}\right)$. The numbering systems for the pyrazolyl carbons are shown in Figure $\mathrm{S} 1$ and are in accord with the method used by Trofimenko. ${ }^{3}$

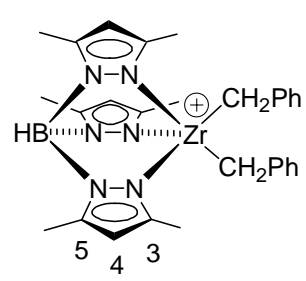

2

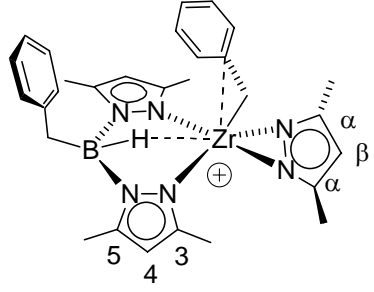

3

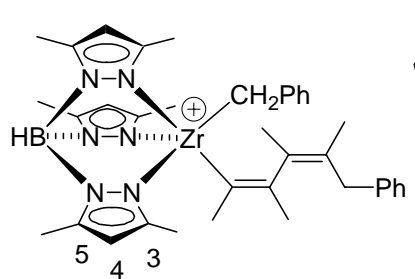

4

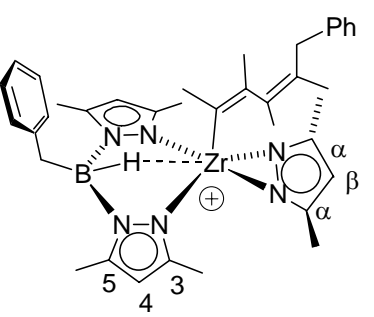

5

Figure S1. The numbering systems for the pyrazolyl carbons.

Tp* $\mathbf{Z r}\left(\mathbf{C H}_{2} \mathbf{P h}\right)_{3}$ (1). A flask was charged with KTp* (738mg, 2.19 mmol) and tetrabenzylzirconium $(1.00 \mathrm{~g}, 2.19 \mathrm{mmol})$. Methylene chloride $(60 \mathrm{~mL})$ was added by

\footnotetext{
${ }^{3}$ Trofimenko, S. Scorpionates: The Coordination Chemistry of Polypyrazolylborate Ligands; Imperial College Press: London, 1999, pp 5-9.
} 
vacuum transfer at $-78{ }^{\circ} \mathrm{C}$. The mixture was stirred at $-78{ }^{\circ} \mathrm{C}$ for 30 min, warmed to 0 ${ }^{\circ} \mathrm{C}$ and stirred for $5 \mathrm{~min}$. The yellow mixture was concentrated to ca. $20 \mathrm{~mL}$ at $0{ }^{\circ} \mathrm{C}$. Hexanes $(40 \mathrm{~mL})$ was added by vacuum transfer at $-78^{\circ} \mathrm{C}$. The resulting yellow mixture was filtered through a frit. The volatiles were removed from the filtrate under vacuum at $0{ }^{\circ} \mathrm{C}$. The remaining yellow solid was washed with cold pentane $(3 \times 10 \mathrm{~mL})$. The solid was dried under vacuum (987 mg, $68 \%$ ). This material, which was spectroscopically pure, was recrystallized from pentane at $-35{ }^{\circ} \mathrm{C}$ yielding X-ray quality crystals. ${ }^{1} \mathrm{H}$ NMR $\left(\mathrm{CD}_{2} \mathrm{Cl}_{2}\right): \delta 7.01(\mathrm{t}, J=7.7,6 \mathrm{H}, m-\mathrm{Ph}), 6.78(\mathrm{t}, J=7.4,3 \mathrm{H}, p-\mathrm{Ph}), 6.66(\mathrm{~d}, J=7.1,6 \mathrm{H}, o-$ Ph), 5.79 (s, 3H, 4-pz), 4.82 (br, 1H, BH), 3.03 (s, 6H, $\mathrm{CH}_{2}$ ), 2.45 (s, 9H, 3- or 5-Me $2 \mathrm{pz}$ ), 2.23 (s, 9H, 3- or 5-Me $2 \mathrm{pz}) .{ }^{13} \mathrm{C}\left\{{ }^{1} \mathrm{H}\right\}$ NMR $\left(\mathrm{CD}_{2} \mathrm{Cl}_{2}\right): \delta 152.8$ (pz, 3- or 5-C), 150.0 (ipso-Ph), 146.3 (3- or 5-C, pz), 128.1, 126.7, $121.6(p-\mathrm{Ph}), 107.6(\mathrm{pz}, 4-\mathrm{C}), 86.5\left(J_{\mathrm{CH}}=\right.$ 113, $\left.\mathrm{ZrCH}_{2}\right), 15.5$ (3- or 5-Me $\left.2 \mathrm{pz}\right), 13.2$ (3- or 5-Me $\left.2 \mathrm{pz}\right) .{ }^{11} \mathrm{~B} \mathrm{NMR}\left(\mathrm{CD}_{2} \mathrm{Cl}_{2}\right): \delta$-9.6. Anal. Calcd for $\mathrm{C}_{36} \mathrm{H}_{43} \mathrm{BN}_{6} \mathrm{Zr}$ : C, 65.33; H, 6.55; N, 12.70. Found: C, 65.39; H, 6.47; $\mathrm{N}, 12.81$.

Generation of $\left[\mathbf{T p}^{*} \mathrm{Zr}\left(\mathbf{C H}_{2} \mathbf{P h}\right)_{2}\right]\left[\mathbf{B}\left(\mathrm{C}_{6} \mathbf{F}_{5}\right)_{4}\right]$ (2). A valved NMR tube was charged with $1(7.2 \mathrm{mg}, 0.011 \mathrm{mmol})$ and $\left[\mathrm{Ph}_{3} \mathrm{C}\right]\left[\left[\mathrm{B}\left(\mathrm{C}_{6} \mathrm{~F}_{5}\right)_{4}\right](10 \mathrm{mg}, 0.011 \mathrm{mmol})\right.$, and $\mathrm{CD}_{2} \mathrm{Cl}_{2}(0.6 \mathrm{~mL})$ was added by vacuum transfer at $-78^{\circ} \mathrm{C}$. The tube was shaken at this temperature to give an orange solution and then was placed in an NMR probe that had been precooled to $-60{ }^{\circ} \mathrm{C}$. A ${ }^{1} \mathrm{H}$ NMR spectrum was obtained and showed that $\left[\mathrm{Tp} * \mathrm{Zr}\left(\mathrm{CH}_{2} \mathrm{Ph}\right)_{2}\right]\left[\mathrm{B}\left(\mathrm{C}_{6} \mathrm{~F}_{5}\right)_{4}\right]$ had formed quantitatively. ${ }^{1} \mathrm{H}$ NMR $\left(\mathrm{CD}_{2} \mathrm{Cl}_{2},-60{ }^{\circ} \mathrm{C}\right): \delta$ 7.23-7.16 (m, 4H, $m-\mathrm{Ph}$, obscured with $\left.\mathrm{Ph}_{3} \mathrm{CCH}_{2} \mathrm{Ph}\right), 7.01$ (t, $J=7.3,2 \mathrm{H}, p-\mathrm{Ph}$, overlapped with $\mathrm{Ph}_{3} \mathrm{CCH}_{2} \mathrm{Ph}$ ), 6.50 (d, $J=7.6,4 \mathrm{H}, o-\mathrm{Ph}$ ), 5.95 (s, 3H, 4-pz), 4.51 (br s, $1 \mathrm{H}, \mathrm{BH}), 2.92\left(\mathrm{~s}, 4 \mathrm{H}, \mathrm{CH}_{2}\right), 2.33$ (s, 9H, 3- or 5-Me $\left.2 \mathrm{pz}\right), 2.18$ (s, 9H, 3- or 5-Me $2 \mathrm{pz}$ ). 
${ }^{13} \mathrm{C}\left\{{ }^{1} \mathrm{H}\right\}$ NMR $\left(\mathrm{CD}_{2} \mathrm{Cl}_{2},-60{ }^{\circ} \mathrm{C}\right): \delta 152.3,149.3,139.2,130.7,127.7,126.9,108.7$ (pz, 4C), $92.1\left({ }^{1} J_{\mathrm{CH}}=127, \mathrm{ZrCH}_{2}\right), 14.9$ (3- or 5-Me $\left.2 \mathrm{pz}\right), 13.0$ (3- or 5-Me $\left.2 \mathrm{pz}\right) .{ }^{11} \mathrm{~B} \mathrm{NMR}$ $\left(\mathrm{CD}_{2} \mathrm{Cl}_{2},-60^{\circ} \mathrm{C}\right): \delta-8.8$.

\section{Generation of $\left[\left\{\left(\mathrm{PhCH}_{2}\right)(\mathrm{H}) \mathrm{B}\left(\mu-\mathrm{Me}_{2} \mathrm{pz}\right)_{2}\right\} \mathrm{Zr}\left(\eta^{2}-\mathrm{Me}_{2} \mathrm{pz}\right)\left(\mathrm{CH}_{2} \mathrm{Ph}\right)\right]\left[\mathrm{B}\left(\mathrm{C}_{6} \mathrm{~F}_{5}\right)_{4}\right]$}

(3). A solution of 2 in $\mathrm{CD}_{2} \mathrm{Cl}_{2}(0.62 \mathrm{~mL})$ at $-60{ }^{\circ} \mathrm{C}$ was prepared as described above. The NMR probe was warmed to $0{ }^{\circ} \mathrm{C}$ for $10 \mathrm{~min}$ and cooled back to $-60^{\circ} \mathrm{C}$. A ${ }^{1} \mathrm{H}$ NMR spectrum was obtained and showed that $\mathbf{3}$ had formed quantitatively. ${ }^{1} \mathrm{H} \mathrm{NMR}\left(\mathrm{CD}_{2} \mathrm{Cl}_{2}\right.$, $\left.-94{ }^{\circ} \mathrm{C}\right): \delta 7.33-7.30\left(\mathrm{~m}, 4 \mathrm{H}, o-\right.$ and $\left.m-\mathrm{BCH}_{2} \mathrm{Ph}\right), 7.20-7.15\left(\mathrm{~m}, 3 \mathrm{H}, p-\mathrm{CH}_{2} \mathrm{Ph}\right.$ and $m-$ $\mathrm{ZrCH}_{2} \mathrm{Ph}$, obscured with $\left.\mathrm{Ph}_{3} \mathrm{CCH}_{2} \mathrm{Ph}\right), 7.02-6.96\left(\mathrm{~m}, 1 \mathrm{H}, p-\mathrm{CH}_{2} \mathrm{Ph}\right.$, obscured with $\mathrm{Ph}_{3} \mathrm{CCH}_{2} \mathrm{Ph}$ ), 6.80 (s, $1 \mathrm{H}, \beta$-pz, $\eta^{2}-\mathrm{Me}_{2} \mathrm{pz}$ ), 6.65 (br s, $1 \mathrm{H}, o-\mathrm{ZrCH}_{2} \mathrm{Ph}$ ), 6.16 (br s, $1 \mathrm{H}, o-$ $\mathrm{ZrCH}_{2} \mathrm{Ph}$ ), 5.68 (s, 2H, 4-pz), 4.53 (br s, 1H, BH), 2.96 (br s, 2H, $\mathrm{ZrCH}_{2}$ ), 2.85 (br s, 2H, $\mathrm{BCH}_{2}$ ), 2.38 (s, 6H, 5-Me $2 \mathrm{pz}$ ), 2.35 (s, 6H, $\eta^{2}-\mathrm{Me}_{2} \mathrm{pz}$ ), 1.83 (br s, 3H, 3-Me $\mathrm{pz}$ ), 1.25 (br s, 3H, 3-Me 2 pz). ${ }^{1} \mathrm{H}$ NMR $\left(\mathrm{CD}_{2} \mathrm{Cl}_{2},-60{ }^{\circ} \mathrm{C}\right): \delta 7.35-7.31$ (m, $4 \mathrm{H}, o-$ and $\left.m-\mathrm{BCH}_{2} \mathrm{Ph}\right)$, 7.21-7.14 (m, 3H, $p-\mathrm{CH}_{2} \mathrm{Ph}$ and $m-\mathrm{ZrCH}_{2} \mathrm{Ph}$, obscured with $\left.\mathrm{Ph}_{3} \mathrm{CCH}_{2} \mathrm{Ph}\right), 6.99(\mathrm{~m}, 1 \mathrm{H}, p$ $\mathrm{CH}_{2} \mathrm{Ph}$, obscured with $\mathrm{Ph}_{3} \mathrm{CCH}_{2} \mathrm{Ph}$ ), 6.83 (s, $1 \mathrm{H}, \beta-\mathrm{pz}, \eta^{2}-\mathrm{Me}_{2} \mathrm{pz}$ ), 6.36 (br d, $J=5.7,2 \mathrm{H}$, $o-\mathrm{ZrCH}_{2} \mathrm{Ph}$ ), 5.73 (s, 2H, 4-pz), 4.77 (br s, 1H, BH), 3.02 (br s, 2H, $\mathrm{ZrCH}_{2}$ ), 2.90 (br s, $2 \mathrm{H}, \mathrm{BCH}_{2}$ ), 2.42 (s, 6H, 5-Me $\left.2 \mathrm{pz}\right), 2.38$ (s, 6H, $\left.\eta^{2}-M e_{2} \mathrm{pz}\right), 1.61$ (br s, 6H, 3-Me $\left.2 \mathrm{pz}\right) .{ }^{1} \mathrm{H}$ NMR assignments were made based on NOESY experiments. NOESY $\left(\mathrm{CD}_{2} \mathrm{Cl}_{2},-35^{\circ} \mathrm{C}\right.$, $\delta \& \delta$ ): major cross peaks $7.34 \& 2.94,7.34 \& 2.46,6.85 \& 2.41,6.33 \& 3.07,5.77 \&$ 2.46, $5.77 \& 1.67,2.94 \& 2.46,2.41 \& 1.67 .{ }^{13} \mathrm{C}\left\{{ }^{1} \mathrm{H}\right\}$ NMR $\left(\mathrm{CD}_{2} \mathrm{Cl}_{2},-20{ }^{\circ} \mathrm{C}\right): \delta 151.5$, $151.4,148.4,142.5,135.5,133.2,130.3,129.4,129.0,128.4,125.5,122.8\left({ }^{1} J_{\mathrm{CH}}=173, \beta-\right.$ pz), $108.6\left({ }^{1} J_{\mathrm{CH}}=177.8,4-\mathrm{pz}\right), 74.8\left({ }^{1} J_{\mathrm{CH}}=144, \mathrm{ZrCH}_{2}\right), 24.6\left(\mathrm{br}, \mathrm{BCH}_{2}\right), 13.2,13.1$ (two Me resonances overlapped). The assignment for $\mathrm{BCH}_{2}$ was based on an HMQC 
experiments. $\mathrm{HMQC}\left(\mathrm{CD}_{2} \mathrm{Cl}_{2},-20{ }^{\circ} \mathrm{C},{ }^{13} \mathrm{C} \delta \&{ }^{1} \mathrm{H} \delta\right): 74.8 \& 3.07,24.6 \& 2.94 .{ }^{11} \mathrm{~B}$ $\operatorname{NMR}\left(\mathrm{CD}_{2} \mathrm{Cl}_{2},-60^{\circ} \mathrm{C}\right): \delta-1.3$.
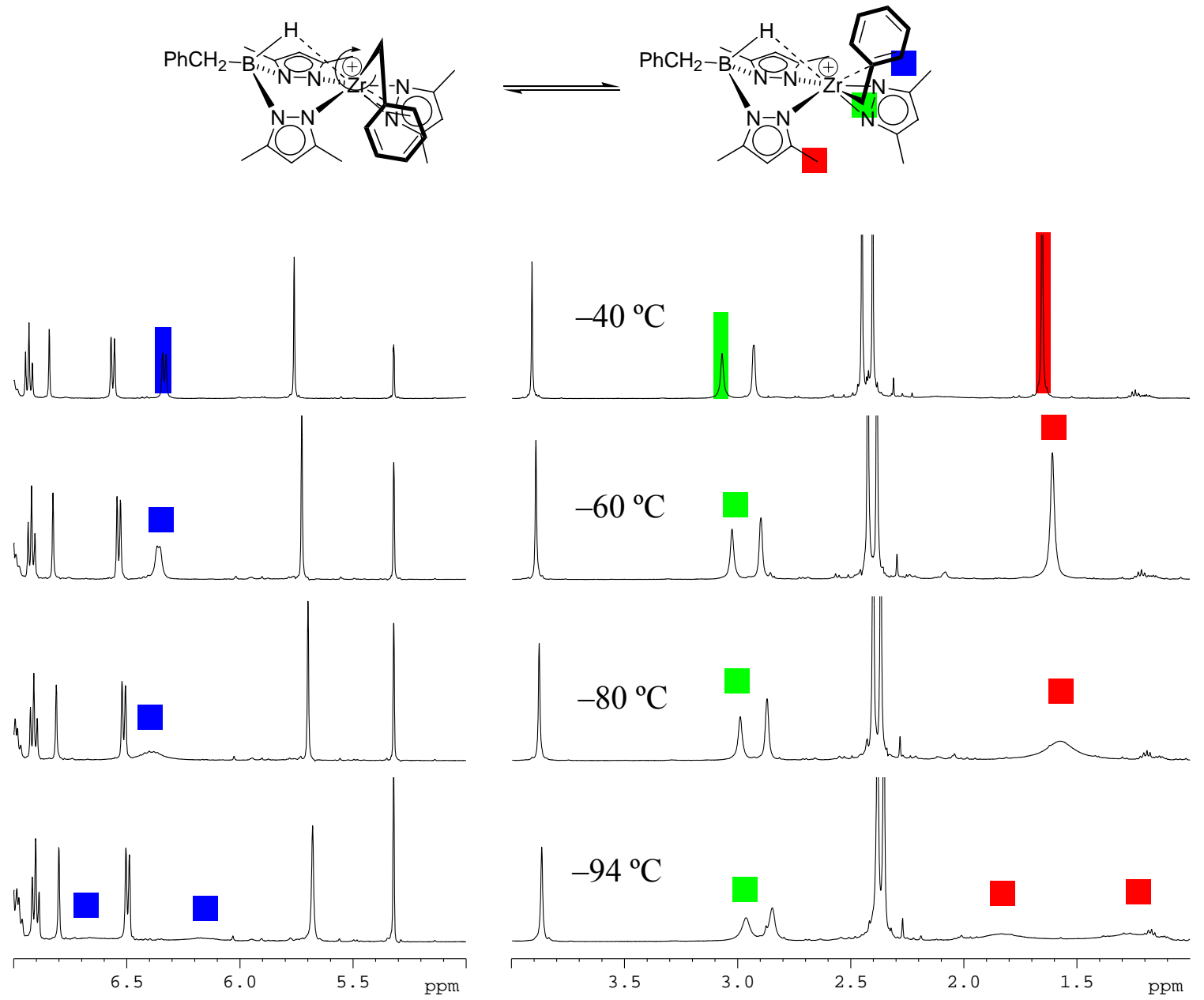

Figure S2. VT NMR spectra of 3

X-ray quality crystals of 3 were obtained from toluene mixture at $-80{ }^{\circ} \mathrm{C}$. The detailed procedure is described below. A valved NMR tube was charged with 1 (5.0 mg, $0.0076 \mathrm{mmol})$ and $\left[\mathrm{Ph}_{3} \mathrm{C}\right]\left[\left[\mathrm{B}\left(\mathrm{C}_{6} \mathrm{~F}_{5}\right)_{4}\right](7.0 \mathrm{~g}, 0.0076 \mathrm{mmol})\right.$, and toluene- $d_{8}(0.7 \mathrm{~mL})$ was added by vacuum transfer at $-78{ }^{\circ} \mathrm{C}$. The tube was shaken at this temperature and an orange oil formed on the bottom of the tube. The tube was warmed to $23{ }^{\circ} \mathrm{C}$ for $20 \mathrm{~min}$ 
and kept at $-80^{\circ} \mathrm{C}$. After 3 months, yellow X-ray quality crystals formed on the bottom of the tube.

\section{$\left[\left\{\left(\mathrm{PhCH}_{2}\right)(\mathrm{H}) \mathrm{B}\left(\mu-\mathrm{Me}_{2} \mathrm{pz}\right)_{2}\right\} \mathrm{Zr}\left(\eta^{2}-\mathrm{Me}_{2} \mathrm{pz}\right)\left(\mathrm{CH}_{2} \mathrm{Ph}\right)\left(\mathrm{PMe}_{3}\right)\right]\left[\mathrm{B}\left(\mathrm{C}_{6} \mathrm{~F}_{5}\right)_{4}\right]\left(3 \cdot \mathrm{PMe}_{3}\right)$.}

A flask was charged with $1(100 \mathrm{mg}, 0.15 \mathrm{mmol})$ and $\left[\mathrm{Ph}_{3} \mathrm{C}\right]\left[\mathrm{B}\left(\mathrm{C}_{6} \mathrm{~F}_{5}\right)_{4}\right](139 \mathrm{mg}, 0.15$ mmol). Methylene chloride $(8 \mathrm{~mL})$ was added by vacuum transfer at $-78^{\circ} \mathrm{C}$. The mixture was warmed to $0{ }^{\circ} \mathrm{C}$ and stirred for $15 \mathrm{~min}$ yielding a yellow solution. The solution was frozen at $-196{ }^{\circ} \mathrm{C}$, and $\mathrm{PMe}_{3}(0.45 \mathrm{mmol}, 3$ equiv) was added by vacuum transfer from a calibrated gas bulb. The mixture was warmed to $23{ }^{\circ} \mathrm{C}$ yielding an orange solution. The solution was concentrated to ca. $3 \mathrm{~mL}$ under vacuum at $0{ }^{\circ} \mathrm{C}$. Hexanes $(10$ $\mathrm{mL}$ ) was added by vacuum transfer at $-78{ }^{\circ} \mathrm{C}$. The mixture was stirred at $23{ }^{\circ} \mathrm{C}$ for 5 min and an orange oil formed on the bottom of the flask. The oil was separated from pale yellow supernatant and dried under vacuum to yield an orange solid (160 mg, $81 \%)$. This material, which was spectroscopically pure, was recrystallized from a mixture of hexanes layered on $\mathrm{CH}_{2} \mathrm{Cl}_{2}$ yielding X-ray quality crystals. ${ }^{1} \mathrm{H} \mathrm{NMR}\left(\mathrm{CD}_{2} \mathrm{Cl}_{2}\right): \delta 7.37(\mathrm{t}$, $J=7.5,2 \mathrm{H}, m-\mathrm{Ph}), 7.31(\mathrm{~d}, J=7.4,2 \mathrm{H}, o-\mathrm{Ph}), 7.22(\mathrm{t}, J=7.2,1 \mathrm{H}, p-\mathrm{Ph}), 7.03(\mathrm{t}, J=7.4$, 2H, $m-\mathrm{Ph}), 6.96(\mathrm{t}, J=7.3,1 \mathrm{H}, p-\mathrm{Ph}), 6.77(\mathrm{~s}, 1 \mathrm{H}, 4-\mathrm{pz}), 6.14(\mathrm{~d}, J=7.4,2 \mathrm{H}, o-$ $\mathrm{ZrCH}_{2} \mathrm{Ph}$ ), 5.79 (s, 1H, 4-pz), 5.68 (s, 1H, 4-pz), 3.88 (br s, 1H, BH), 3.17 (dd, J= 15.0, 4.3; $\left.1 \mathrm{H}, \mathrm{BCH}_{2}\right), 2.75\left(\mathrm{~d},{ }^{3} J_{\mathrm{PH}}=6.3,2 \mathrm{H}, \mathrm{ZrCH}_{2}\right.$, assignment confirmed by ${ }^{1} \mathrm{H}\left\{{ }^{31} \mathrm{P}\right\}$ NMR), $2.71\left(\mathrm{~d}, J=15.3,1 \mathrm{H}, \mathrm{BCH}_{2}\right), 2.47(\mathrm{~s}, 3 \mathrm{H}, 3-$ or 5-Me $2 \mathrm{pz}), 2.44$ (s, 3H, 3- or 5$\left.M e_{2} \mathrm{pz}\right), 2.37$ (s, 6H, $\left.\eta^{2}-M e_{2} \mathrm{pz}\right), 1.77$ (s, 3H, 3- or 5-Me $\left.2 \mathrm{pz}\right), 1.27$ (s, 3H, 3- or 5-Me $\left.2 \mathrm{pz}\right)$, $1.24\left(\mathrm{~d}, 9 \mathrm{H},{ }^{2} J_{\mathrm{PH}}=7.9, \mathrm{PMe}_{3}\right) .{ }^{13} \mathrm{C}\left\{{ }^{1} \mathrm{H}\right\} \mathrm{NMR}\left(\mathrm{CD}_{2} \mathrm{Cl}_{2}\right): \delta 151.3,150.8,150.2,148.10$, 148.06, 142.5, $132.9\left({ }^{3} J_{\mathrm{PC}}=5\right.$, ipso- $\left.\mathrm{ZrCH}_{2} \mathrm{Ph}\right), 132.4,131.2,129.1,129.0,128.5,125.9$, 122.0, 108.7, 108.2, $69.9\left({ }^{2} J_{\mathrm{PC}}=5, J_{\mathrm{CH}}=138, \mathrm{ZCCH}_{2}\right), 25.2\left(\mathrm{br}, \mathrm{BCH}_{2}\right.$, the assignment 
was based on an HMQC experiment), $14.7\left(\mathrm{~d},{ }^{1} J_{\mathrm{PC}}=19, \mathrm{PMe}_{3}\right), 14.5,13.62,13.60,13.0$, 11.9. HMQC $\left(\mathrm{CD}_{2} \mathrm{Cl}_{2},{ }^{13} \mathrm{C} \delta \&{ }^{1} \mathrm{H} \delta\right): 25.2 \& 3.17,69.9 \& 2.75,25.2 \& 2.71 .{ }^{11} \mathrm{~B}$ NMR $\left(\mathrm{CD}_{2} \mathrm{Cl}_{2}\right): \delta-2.0 .{ }^{31} \mathrm{P}$ NMR $\left(\mathrm{CD}_{2} \mathrm{Cl}_{2}\right): \delta$ 18.8. Anal. Calcd for $\mathrm{C}_{56} \mathrm{H}_{45} \mathrm{~B}_{2} \mathrm{~F}_{20} \mathrm{~N}_{6} \mathrm{PZr}$ : C, 50.73; H, 3.42; N, 6.34. Found: C, 50.48; H, 3.60; N, 6.26.

Generation of $\left[\mathrm{Tp} * \mathrm{Zr}\left(\mathrm{CMe}=\mathrm{CMeCMe}=\mathrm{CMeCH}_{2} \mathrm{Ph}\right)\left(\mathrm{CH}_{2} \mathrm{Ph}\right)\right]\left[\mathrm{B}\left(\mathrm{C}_{6} \mathrm{~F}_{5}\right)_{4}\right]$ (4). A solution of $2(0.011 \mathrm{mmol})$ in $\mathrm{CD}_{2} \mathrm{Cl}_{2}(0.59 \mathrm{~mL})$ at $-60^{\circ} \mathrm{C}$ was prepared as described above. 2-Butyne ( $0.065 \mathrm{mmol}, 6$ equiv) was added by vacuum transfer from a calibrated gas bulb at $-196{ }^{\circ} \mathrm{C}$. The tube was warmed to $-78{ }^{\circ} \mathrm{C}$, shaken vigorously at this temperature for ca. 1 min to produce a red solution, and placed in an NMR probe that had been precooled to $-60^{\circ} \mathrm{C}$. NMR spectra showed that 4 had formed quantitatively. Free unreacted 2-butyne (4 equiv) was observed in the spectra. ${ }^{1} \mathrm{H} \mathrm{NMR}\left(\mathrm{CD}_{2} \mathrm{Cl}_{2},-60{ }^{\circ} \mathrm{C}\right): \delta$ $7.27(\mathrm{t}, J=7.4,2 \mathrm{H}, m-\mathrm{Ph}), 7.22-7.11(\mathrm{~m}, 5 \mathrm{H}, o-, m-$ and $p$-Ph, obscured with $\left.\mathrm{Ph}_{3} \mathrm{CCH}_{2} \mathrm{Ph}\right), 6.84$ (t, $\left.J=7.4,1 \mathrm{H}, p-\mathrm{Ph}\right), 6.17$ (d, $\left.J=7.5,2 \mathrm{H}, o-\mathrm{ZrCH}_{2} \mathrm{Ph}\right), 6.04(\mathrm{~s}, 1 \mathrm{H}, 4-$ pz), 5.95 (s, 1H, 4-pz), 5.77 (s, 1H, 4-pz), 4.45 (br s, 1H, BH), 3.81 (d, $J=15.1,1 \mathrm{H}$, $\mathrm{CMeCH}_{2} \mathrm{Ph}$ ), 3.34 (d, $\left.J=15.0,1 \mathrm{H}, \mathrm{CMeCH}_{2} \mathrm{Ph}\right), 2.94$ (d, $\left.J=8.3,1 \mathrm{H}, \mathrm{ZrCH}_{2} \mathrm{Ph}\right), 2.80$ (d, $\left.J=8.3,1 \mathrm{H}, \mathrm{ZrCH}_{2} \mathrm{Ph}\right), 2.42(\mathrm{~s}, 3 \mathrm{H}, \mathrm{Me}), 2.38(\mathrm{~s}, 3 \mathrm{H}, \mathrm{Me}), 2.24$ (s, 6H, overlapped two different Me's), 2.16 (s, 3H, Me), 2.14 (s, 3H, Me), 2.10 (s, 3H, Me), 1.90 (s, 3H, Me), $1.87(\mathrm{~s}, 3 \mathrm{H}, \mathrm{Me}), 1.58(\mathrm{~s}, 3 \mathrm{H}, \mathrm{Me}) .{ }^{13} \mathrm{C}\left\{{ }^{1} \mathrm{H}\right\} \mathrm{NMR}\left(\mathrm{CD}_{2} \mathrm{Cl}_{2},-60{ }^{\circ} \mathrm{C}\right): \delta 201.0(\mathrm{ZrCMe}=)$, $154.2,151.6,151.5,150.9,149.6,147.21,147.15,139.1,137.2,135.6,132.4,130.5$, $129.0,128.2,128.1,128.0,126.0,108.9$ (4-pz), $108.6(4-p z), 106.9(4-p z), 78.2\left({ }^{1} J_{\mathrm{CH}}=\right.$ $\left.140, \mathrm{ZrCH}_{2}\right), 41.1\left({ }^{1} J_{\mathrm{CH}}=123, \mathrm{CMeCH}_{2} \mathrm{Ph}\right), 21.8,21.3,17.3,15.1,14.6,13.4,13.0,12.6$, 12.5, 5.4. ${ }^{11} \mathrm{~B}$ NMR $\left(\mathrm{CD}_{2} \mathrm{Cl}_{2},-60{ }^{\circ} \mathrm{C}\right): \delta-8.0$. 
Hydrolysis of 4. A solution of $4(0.011 \mathrm{mmol})$ in $\mathrm{CD}_{2} \mathrm{Cl}_{2}(0.59 \mathrm{~mL})$ was prepared as described above, and cooled to $-78{ }^{\circ} \mathrm{C}$. A drop of water was added. The mixture was slowly warmed to room temperature while the tube was vigorously shaken. The volatiles were vacuum transferred to another valved NMR tube. NMR and GC-MS showed that toluene and 2-butyne were present in the volatiles. The white solid that remained after removal of the volatiles was dissolved in $\mathrm{CH}_{2} \mathrm{Cl}_{2}$ and filtered through alumina. The alumina was washed with pentane $(2 \mathrm{~mL})$. The filtrate and wash were combined and evaporated to dryness yielding a white wax. The wax was extracted with pentane. The pentane extract was dried under vacuum and dissolved in $\mathrm{CD}_{2} \mathrm{Cl}_{2}$ for $\mathrm{NMR}$ and GC-MS analysis. ${ }^{1} \mathrm{H}$ NMR and GC-MS showed that the extract contained $\mathrm{Ph}_{3} \mathrm{CCH}_{2} \mathrm{Ph}$ and $(Z, E)-2,3,4$-trimethyl-1-phenyl-2,4-hexadiene. The same procedure was repeated except that $\mathrm{D}_{2} \mathrm{O}$ was used instead of water. The product from deuterolysis contained $(Z, E)$-5-deutero-2,3,4-trimethyl-1-phenyl-2,4-hexadiene.

Data for $(Z, E)-2,3,4$-trimethyl-1-phenyl-2,4-hexadiene: ${ }^{1} \mathrm{H}$ NMR $\left(\mathrm{CD}_{2} \mathrm{Cl}_{2}\right): \delta$ 7.29-7.12 (m, 5H, o-, $m$ - and $p$-Ph, obscured with $\mathrm{Ph}_{3} \mathrm{CCH}_{2} \mathrm{Ph}$ ), 5.23 (qq, $J=6.8,1.5$, $1 \mathrm{H},=\mathrm{CHMe}), 3.40\left(\mathrm{~s}, 2 \mathrm{H}, \mathrm{CH}_{2}\right), 1.75(\mathrm{q}, J=0.8,3 \mathrm{H}), 1.71(\mathrm{dq}, J=1.2,1.2,3 \mathrm{H}$ $\left.\mathrm{MeHC}=\mathrm{CMeCMe}=\mathrm{CMeCH}_{2} \mathrm{Ph}\right), 1.61(\mathrm{dq}, J=6.8,1.2,3 \mathrm{H},=\mathrm{CHMe}), 1.53(\mathrm{q}, J=0.8$, 3H). GC/MS: m/z $200\left(\mathrm{M}^{+}\right)$, 185, 171, 156, 143, 128, 115, 109, 91, 77, 67, 55.

Data for $(Z, E)$-5-deutero-2,3,4-trimethyl-1-phenyl-2,4-hexadiene: ${ }^{1} \mathrm{H} \quad \mathrm{NMR}$ $\left(\mathrm{CD}_{2} \mathrm{Cl}_{2}\right): \delta$ 7.24-7.17 (m, 5H, o-, $m$ - and $p$-Ph, obscured with $\left.\mathrm{Ph}_{3} \mathrm{CCH}_{2} \mathrm{Ph}\right), 3.40(\mathrm{~s}, 2 \mathrm{H}$, $\left.\mathrm{CH}_{2}\right), \quad 1.74(\mathrm{q}, \quad J=1.1,3 \mathrm{H}), 1.71\left(\mathrm{tq},{ }^{4} J_{\mathrm{DH}}=1.4,{ }^{5} J_{\mathrm{HH}}=1.4,3 \mathrm{H}\right.$, $\left.\mathrm{MeDC}=\mathrm{CMeCMe}=\mathrm{CMeCH}_{2} \mathrm{Ph}\right), 1.60($ br $\mathrm{m}, 3 \mathrm{H},=\mathrm{CDMe}), 1.52(\mathrm{q}, J=1.1,3 \mathrm{H})$. GC/MS: m/z $201\left(\mathrm{M}^{+}\right)$, 186, 171, 156, 143, 129, 115, 110, 91, 77, 65. 


\section{Generation of $\left[\left\{\left(\mathrm{PhCH}_{2}\right)(\mathrm{H}) \mathrm{B}\left(\mu-\mathrm{Me}_{2} \mathrm{pz}\right)_{2}\right\} \mathrm{Zr}\left(\mathrm{CMe}=\mathrm{CMeCMe}=\mathrm{CMeCH}_{2} \mathrm{Ph}\right)\right.$}

$\left.\left(\eta^{2}-\mathbf{M e}_{2} \mathbf{p z}\right)\right]\left[\mathbf{B}\left(\mathbf{C}_{6} \mathbf{F}_{5}\right)_{4}\right]$ (5). A solution of $3(21.5 \mathrm{mg}, 0.033 \mathrm{mmol})$ in $\mathrm{CD}_{2} \mathrm{Cl}_{2}(0.64 \mathrm{~mL})$ at $-60{ }^{\circ} \mathrm{C}$ was prepared as described above. 2-Butyne $(0.070 \mathrm{mmol}, 2.17$ equiv) was added by vacuum transfer from a calibrated gas bulb at $-196{ }^{\circ} \mathrm{C}$. The tube was warmed to $23{ }^{\circ} \mathrm{C}$ for 10 min to produce a yellow solution. The ${ }^{1} \mathrm{H}$ NMR spectrum showed that 5 $(0.027 \mathrm{mmol}, 83 \%)$, cis- $\beta$-methylstyrene $(0.0054 \mathrm{mmol}, 17 \%)$ and $\left[\left\{\left(\mathrm{CH}_{2} \mathrm{Ph}\right)(\mathrm{H}) \mathrm{B}(\mu-\right.\right.$ $\left.\left.\left.\mathrm{Me}_{2} \mathrm{pz}\right)_{2}\right\} \mathrm{Zr}\left(\eta^{2}-\mathrm{Me}_{2} \mathrm{pz}\right)\left(\eta^{5}-\mathrm{C}_{5} \mathrm{Me}_{5}\right)\right]\left[\mathrm{B}\left(\mathrm{C}_{6} \mathrm{~F}_{5}\right)_{4}\right]$ (6) (0.0054 mmol, $\left.17 \%\right)$ had formed. These data imply quantitative formation of 5 from the reaction of 3 and 2 equiv of 2butyne followed by fast reaction of 0.17 equiv of 5 with 2-butyne to form 0.17 equiv of 6 and $c i s$ - $\beta$-methylstyrene. Data for 5: ${ }^{1} \mathrm{H}$ NMR $\left(\mathrm{CD}_{2} \mathrm{Cl}_{2}\right): \delta 7.27-6.64(\mathrm{~m}, 8 \mathrm{H}, o-, m$ - and $p$-Ph, obscured with $\mathrm{Ph}_{3} \mathrm{CCH}_{2} \mathrm{Ph}$ ), 6.83 (d, 2H, $\left.J=7.5, o-\mathrm{Ph}\right) 6.82$ (s, $1 \mathrm{H}, \beta$-pz), 5.97 (s, 2H, 4-pz), 5.89 (br s, $1 \mathrm{H}, \mathrm{BH}), 3.14\left(\right.$ br s, $\left.2 \mathrm{H},-\mathrm{CMeCH}_{2} \mathrm{Ph}\right), 3.02(\mathrm{~d}, J=2.5,2 \mathrm{H}$, BCH $\left.H_{2} \mathrm{Ph}\right), 2.58(\mathrm{~s}, 6 \mathrm{H}), 2.49$ (s, 6H), 2.21 (br s, 6H), $1.90(\mathrm{~s}, 3 \mathrm{H}), 1.72(\mathrm{~s}, 3 \mathrm{H}), 1.69$ (s, $3 \mathrm{H}), 1.58$ (br s, $3 \mathrm{H}) .{ }^{13} \mathrm{C}\left\{{ }^{1} \mathrm{H}\right\}$ NMR $\left(\mathrm{CD}_{2} \mathrm{Cl}_{2}\right): \delta 211.2$ (Zr-C), 152.5, 151.3, 149.1, $144.0,142.0,138.3,129.3,129.1,129.0,128.71,128.68,127.3125 .6,125.5,121.5(\beta-$ pz), 109.4 (4-pz), $43.8-\mathrm{CMeCH}_{2} \mathrm{Ph}$ ), 24.9 (br, $\mathrm{BCH}_{2}$ ), 21.4, 20.0, 18.8, 16.3, 13.9, 13.6, 13.5. ${ }^{11} \mathrm{~B}$ NMR $\left(\mathrm{CD}_{2} \mathrm{Cl}_{2}\right): \delta-2.1$.

Generation of $\left[\left\{\left(\mathrm{PhCH}_{2}\right)(\mathrm{H}) \mathrm{B}\left(\mu-\mathrm{Me}_{2} \mathrm{pz}\right)_{2}\right\} \mathrm{Zr}\left(\eta^{2}-\mathrm{Me}_{2} \mathrm{pz}\right)\left(\eta^{5}-\mathrm{C}_{5} \mathrm{Me}_{5}\right)\right]\left[\mathrm{B}\left(\mathrm{C}_{6} \mathrm{~F}_{5}\right)_{4}\right]$ (6). Method A. A solution of $3(0.011 \mathrm{mmol})$ in $\mathrm{CD}_{2} \mathrm{Cl}_{2}(0.61 \mathrm{~mL})$ at $-60{ }^{\circ} \mathrm{C}$ was prepared as described above. 2-Butyne ( $0.065 \mathrm{mmol}, 6$ equiv) was added by vacuum transfer from a calibrated gas bulb at $-196{ }^{\circ} \mathrm{C}$. The tube was warmed to $20^{\circ} \mathrm{C}$ for $10 \mathrm{~min}$ 
to produce a red solution. NMR spectra showed that $\mathbf{6}$ and $c i s-\beta$-methylstyrene ${ }^{4}$ had formed quantitatively. Data for 6: ${ }^{1} \mathrm{H} \mathrm{NMR}\left(\mathrm{CD}_{2} \mathrm{Cl}_{2}\right): \delta 7.19(\mathrm{t}, J=7.4,2 \mathrm{H}, m-\mathrm{Ph}), 7.13$ (t, $J=7.3,1 \mathrm{H}, p-\mathrm{Ph}), 6.75(\mathrm{~d}, J=7.2, o-\mathrm{Ph}), 6.36(\mathrm{~s}, 1 \mathrm{H}, \beta-\mathrm{pz}), 6.04$ (s, 2H, 4-pz), 3.91 (br s, 1H, BH), 2.78 (br d, $\left.J=3.0,2 \mathrm{H}, \mathrm{BCH}_{2}\right), 2.46(\mathrm{~s}, 6 \mathrm{H}), 2.26(\mathrm{~s}, 6 \mathrm{H}), 2.23(\mathrm{~s}, 6 \mathrm{H})$, $1.89(\mathrm{~s}, 15 \mathrm{H}) .{ }^{13} \mathrm{C}\left\{{ }^{1} \mathrm{H}\right\} \operatorname{NMR}\left(\mathrm{CD}_{2} \mathrm{Cl}_{2}\right): \delta 154.1,149.6,147.8,141.8,129.1,128.9$, 128.6, 125.7, 120.6, 110.6, $24.8\left(\mathrm{br}, \mathrm{BCH}_{2}\right), 14.9,13.7,13.3,12.2\left(\mathrm{C}_{5} M e_{5}\right) .{ }^{11} \mathrm{~B} \mathrm{NMR}$ $\left(\mathrm{CD}_{2} \mathrm{Cl}_{2}\right): \delta-2.7$

Method B. A mixture of 5 (0.027 mmol, 0.83 equiv), cis- $\beta$-methylstyrene (0.0054 mmol, 0.17 equiv) and $6\left(0.0054 \mathrm{mmol}, 0.17\right.$ equiv) at $23{ }^{\circ} \mathrm{C}$ was prepared as described above. Additional 2-butyne (0.049 mmol, 1.5 equiv) was added by vacuum transfer from a calibrated gas bulb at $-196{ }^{\circ} \mathrm{C}$. The tube was warmed to $23{ }^{\circ} \mathrm{C}$ for $10 \mathrm{~min}$ to produce a red solution. NMR spectra showed that $\mathbf{6}$ and cis- $\beta$-methylstyrene had formed quantitatively. The compound was identical in all respects to that prepared by method A.

Independent generation of 6 . A solution of $3(0.0076 \mathrm{mmol})$ in $\mathrm{CD}_{2} \mathrm{Cl}_{2}(0.59$ $\mathrm{mL})$ at $\quad 0 \quad{ }^{\circ} \mathrm{C}$ was prepared as described above. A solution of $1,2,3,4,5-$ pentamethylcyclopentadiene $\left(30.5 \mu\right.$ of $0.25 \mathrm{M}$ solution in $\left.\mathrm{CH}_{2} \mathrm{Cl}_{2}, 0.0076 \mathrm{mmol}\right)$ was added by syringe at $23{ }^{\circ} \mathrm{C}$. The ${ }^{1} \mathrm{H}$ NMR spectrum showed that $\mathbf{6}$ and toluene had formed quantitatively.

${ }^{4}$ Data for cis- $\beta$-methylstyrene: ${ }^{1} \mathrm{H}$ NMR $\left(\mathrm{CD}_{2} \mathrm{Cl}_{2}\right): \delta 7.35-7.20(\mathrm{~m}, 5 \mathrm{H}), 6.43$ $(\mathrm{dq}, J=11.7,1.8,1 \mathrm{H}), 5.80(\mathrm{dq}, J=11.6,7.2,1 \mathrm{H}), 1.89(\mathrm{dd}, J=7.1,1.9,3 \mathrm{H}, \mathrm{Me})$. GC/MS: m/z 118, 117(M+1), 116, 115, 103, 91, 77, 65, 58, 51. 
Hydrolysis of 5. The same procedure for hydrolysis of $\mathbf{4}$ was used for hydrolysis of 5. Hydrolysis of 5 produced (Z,E)-2,3,4-Trimethyl-1-phenyl-2,4-hexadiene and deuterolysis produced (Z,E)-5-deutero-2,3,4-trimethyl-1-phenyl-2,4-hexadiene.

$\left\{\left(\mathrm{PhCH}_{2}\right)(\mathrm{H}) \mathrm{B}\left(\mu-\mathrm{Me}_{2} \mathrm{pz}\right)_{2}\right\} \mathrm{Zr}\left(\eta^{2}-\mathrm{Me}_{2} \mathrm{pz}\right)\left(\eta^{5}-\mathrm{C}_{5} \mathrm{Me}_{5}\right)(\mathrm{Cl}) \quad$ (7). A flask was charged with $1(170 \mathrm{mg}, 0.26 \mathrm{mmol})$ and $\left[\mathrm{Ph}_{3} \mathrm{C}\right]\left[\mathrm{B}\left(\mathrm{C}_{6} \mathrm{~F}_{5}\right)_{4}\right](240 \mathrm{mg}, 0.26 \mathrm{mmol})$. Methylene chloride $(10 \mathrm{~mL})$ was added by vacuum transfer at $-78{ }^{\circ} \mathrm{C}$. The mixture was warmed to $0{ }^{\circ} \mathrm{C}$ and stirred yielding a yellow solution. After $15 \mathrm{~min}$, a solution of 1,2,3,4,5-pentamethylcyclopentadiene $(0.040 \mathrm{ml}, 0.26 \mathrm{mmol})$ in $\mathrm{CH}_{2} \mathrm{Cl}_{2}(10 \mathrm{~mL})$ was added by cannula transfer at $0{ }^{\circ} \mathrm{C}$. The resulting yellow solution was stirred for $10 \mathrm{~min}$ and the volatiles were removed under vacuum yielding a foamy yellow solid. Toluene $(20 \mathrm{~mL})$ was added to the solid and a yellow oil separated. The yellow oil was separated, washed with hexanes $(20 \mathrm{~mL})$, and dried under vacuum to yield a yellow solid, which was identified as 6 by ${ }^{1} \mathrm{H}$ NMR. This material was transferred to another flask, and benzyltributylammonium chloride $(80 \mathrm{mg}, 0.26 \mathrm{mmol}$ ) was added. Methylene chloride $\left(10 \mathrm{~mL}\right.$ ) was added by vacuum transfer at $-78{ }^{\circ} \mathrm{C}$. The solution was warmed to $23{ }^{\circ} \mathrm{C}$ and stirred for $20 \mathrm{~h}$ yielding a colorless solution. The volatiles were removed under vacuum yielding a white solid. The white solid was extracted with hexanes $(5 \times 30 \mathrm{~mL})$. The extracts were combined, concentrated to $30 \mathrm{~mL}$, and cooled to $-35^{\circ} \mathrm{C}$ for 3 days. A crystalline colorless solid formed. The solid was collected by filtration (1st crop, 65.5 mg, $39 \%$ ). This material, which was spectroscopically pure, was recrystallized from saturated methylene chloride solution yielding X-ray quality crystals. The filtrate was concentrated to $10 \mathrm{~mL}$ and kept at $-35^{\circ} \mathrm{C}$. A colorless solid formed after 3 days and was collected by filtration (2nd crop, $22.1 \mathrm{mg}, 13 \%$, total $52 \%) .{ }^{1} \mathrm{H}$ NMR $\left(\mathrm{CD}_{2} \mathrm{Cl}_{2}\right): \delta 7.32$ 
(d, $J=7.4,2 \mathrm{H}, o-\mathrm{Ph}), 7.26(\mathrm{t}, J=7.6,2 \mathrm{H}, m-\mathrm{Ph}), 7.16$ (t, $J=7.2,1 \mathrm{H}, p-\mathrm{Ph}), 5.84(\mathrm{~s}, 1 \mathrm{H}$, 4-pz), 5.66 (s, 1H, 4-pz), 5.32 (s, 1H, 4-pz), 3.18 (dd, $J=14.9,3.4,1 \mathrm{H}, \mathrm{CH}_{2}$ ), 2.68 (d, $J=$ 15.0, 1H, $\left.\mathrm{CH}_{2}\right), 2.61$ (br s, 1H, BH), $2.52(\mathrm{~s}, 3 \mathrm{H}), 2.48(\mathrm{~s}, 3 \mathrm{H}), 2.27$ (s, 3H), $2.26(\mathrm{~s}, 3 \mathrm{H})$, $1.97(\mathrm{~s}, 3 \mathrm{H}), 1.92(\mathrm{~s}, 3 \mathrm{H}), 1.55(\mathrm{~s}, 15 \mathrm{H}) .{ }^{13} \mathrm{C}\left\{{ }^{1} \mathrm{H}\right\} \mathrm{NMR}\left(\mathrm{CD}_{2} \mathrm{Cl}_{2}\right): \delta 151.4,148.5$, 144.4, 144.1, 143.7, 142.6, 141.8, 129.8, 128.8, 125.2, 123.9, 110.7 (4-pz), 109.9 (4-pz), 106.4 (4-pz), 26.4 (br, $\left.\mathrm{BCH}_{2}\right), 15.4,13.7,13.3,13.2,12.9,12.5,11.7\left(\mathrm{C}_{5} \mathrm{Me}_{5}\right) .{ }^{11} \mathrm{~B}$ NMR $\left(\mathrm{CD}_{2} \mathrm{Cl}_{2}\right): \delta$-2.7. Anal. Calcd for $\mathrm{C}_{32} \mathrm{H}_{44} \mathrm{BClN}_{6} \mathrm{Zr}$ : C, 59.11; H, 6.82; N, 12.92. Found: C, 59.27; H, 6.92; N, 12.68 .

Ethylene Polymerization by 2. A solution of $2(10.8 \mu \mathrm{mol})$ in $\mathrm{CD}_{2} \mathrm{Cl}_{2}(0.59 \mathrm{~mL})$ was prepared as described above. Ethylene ( $480 \mu \mathrm{mol}, 44$ equiv) was added by vacuum transfer from a calibrated gas bulb at $-196{ }^{\circ} \mathrm{C}$. The tube was warmed to $-78{ }^{\circ} \mathrm{C}$ and shaken vigorously at this temperature for ca. $10 \mathrm{~min}$ to produce white precipitate. The mixture was quenched with $1 \mathrm{~mL}$ of methanol. The white precipitate was collected by filtration, dissolved in $1,2-\mathrm{C}_{6} \mathrm{D}_{4} \mathrm{Cl}_{2}$ and analyzed by ${ }^{1} \mathrm{H}$ and ${ }^{13} \mathrm{C}$ NMR at $110{ }^{\circ} \mathrm{C} .{ }^{1} \mathrm{H}$ and ${ }^{13} \mathrm{C}$ NMR analysis confirmed the white precipitate was linear polyethylene (Fig. S3 and S4).

Ethylene Polymerization by 3. A solution of $3(7.7 \mu \mathrm{mol})$ in $\mathrm{CD}_{2} \mathrm{Cl}_{2}(0.60 \mathrm{~mL})$ was prepared as described above. Ethylene ( $385 \mu \mathrm{mol}, 50$ equiv) was added by vacuum transfer from a calibrated gas bulb at $-196{ }^{\circ} \mathrm{C}$. The tube was warmed to $-78{ }^{\circ} \mathrm{C}$, shaken vigorously at this temperature for ca. 10 min to produce white precipitate. The mixture was quenched with $1 \mathrm{~mL}$ of methanol. The white precipitate was collected by filtration, dissolved in $1,2-\mathrm{C}_{6} \mathrm{D}_{4} \mathrm{Cl}_{2}$ and analyzed by ${ }^{1} \mathrm{H}$ and ${ }^{13} \mathrm{C}$ NMR at $110{ }^{\circ} \mathrm{C} .{ }^{1} \mathrm{H}$ and ${ }^{13} \mathrm{C}$ NMR analysis confirmed the white precipitate was linear polyethylene (Fig S5 and S6). 

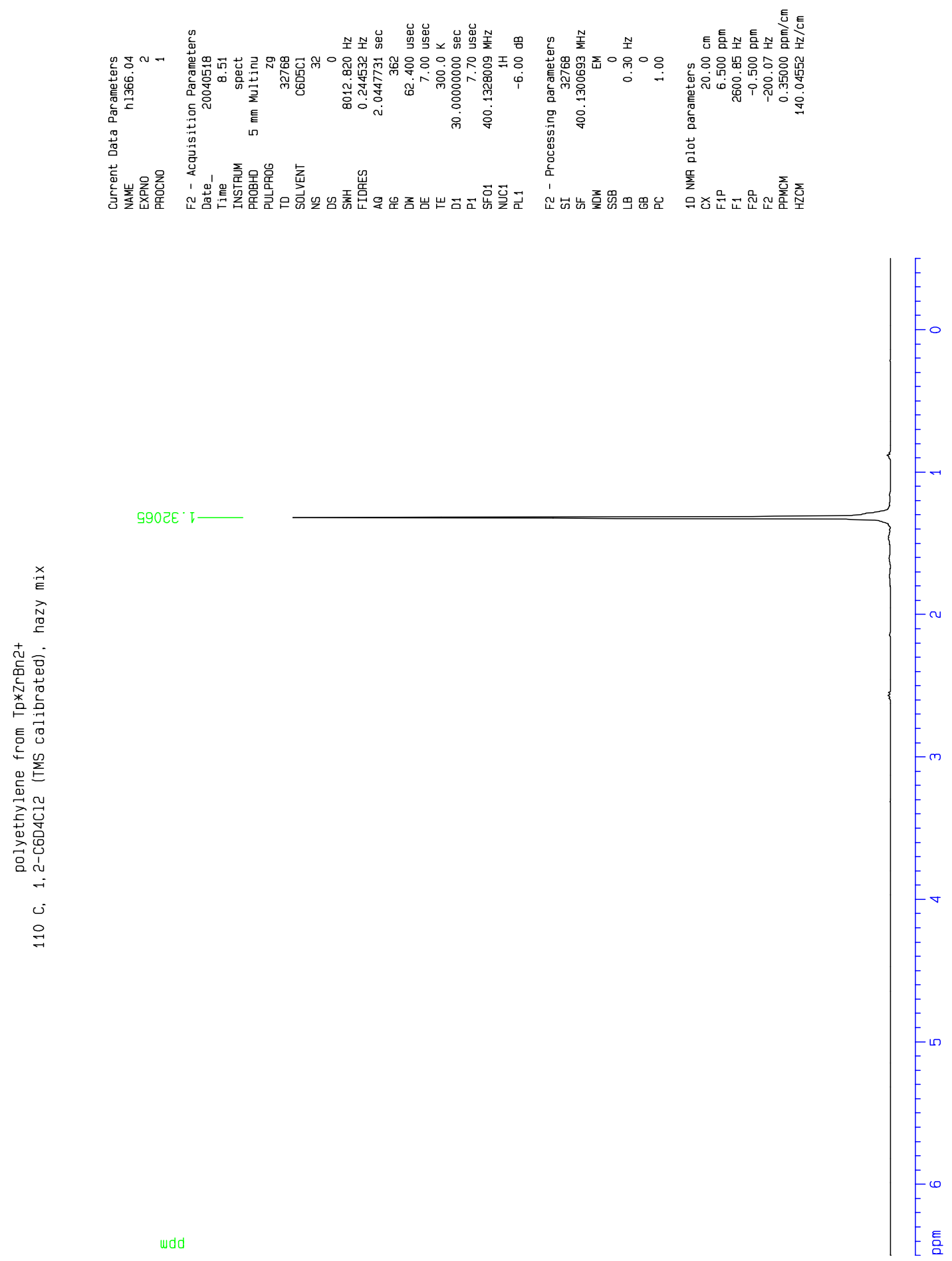

Figure S3. ${ }^{1} \mathrm{H}$ NMR spectrum $\left(110{ }^{\circ} \mathrm{C}\right.$ in $\left.1,2-\mathrm{C}_{6} \mathrm{D}_{4} \mathrm{Cl}_{2}\right)$ of polyethylene produced from 2 at $-78^{\circ} \mathrm{C}$ 

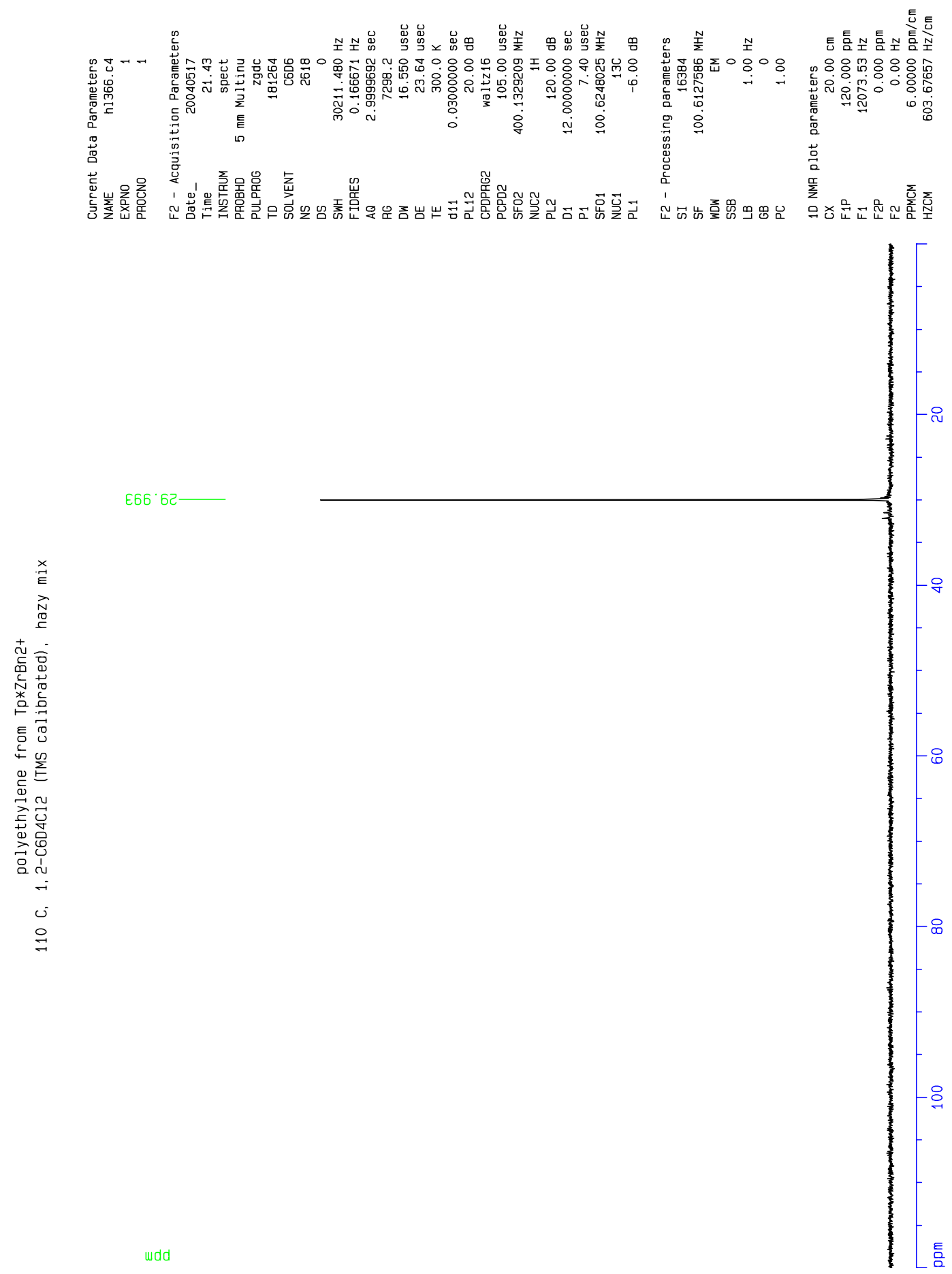

Figure S4. ${ }^{13} \mathrm{C}\left\{{ }^{1} \mathrm{H}\right\}$ NMR spectrum $\left(110{ }^{\circ} \mathrm{C}\right.$ in $\left.1,2-\mathrm{C}_{6} \mathrm{D}_{4} \mathrm{Cl}_{2}\right)$ of polyethylene produced from 2 at $-78^{\circ} \mathrm{C}$ 

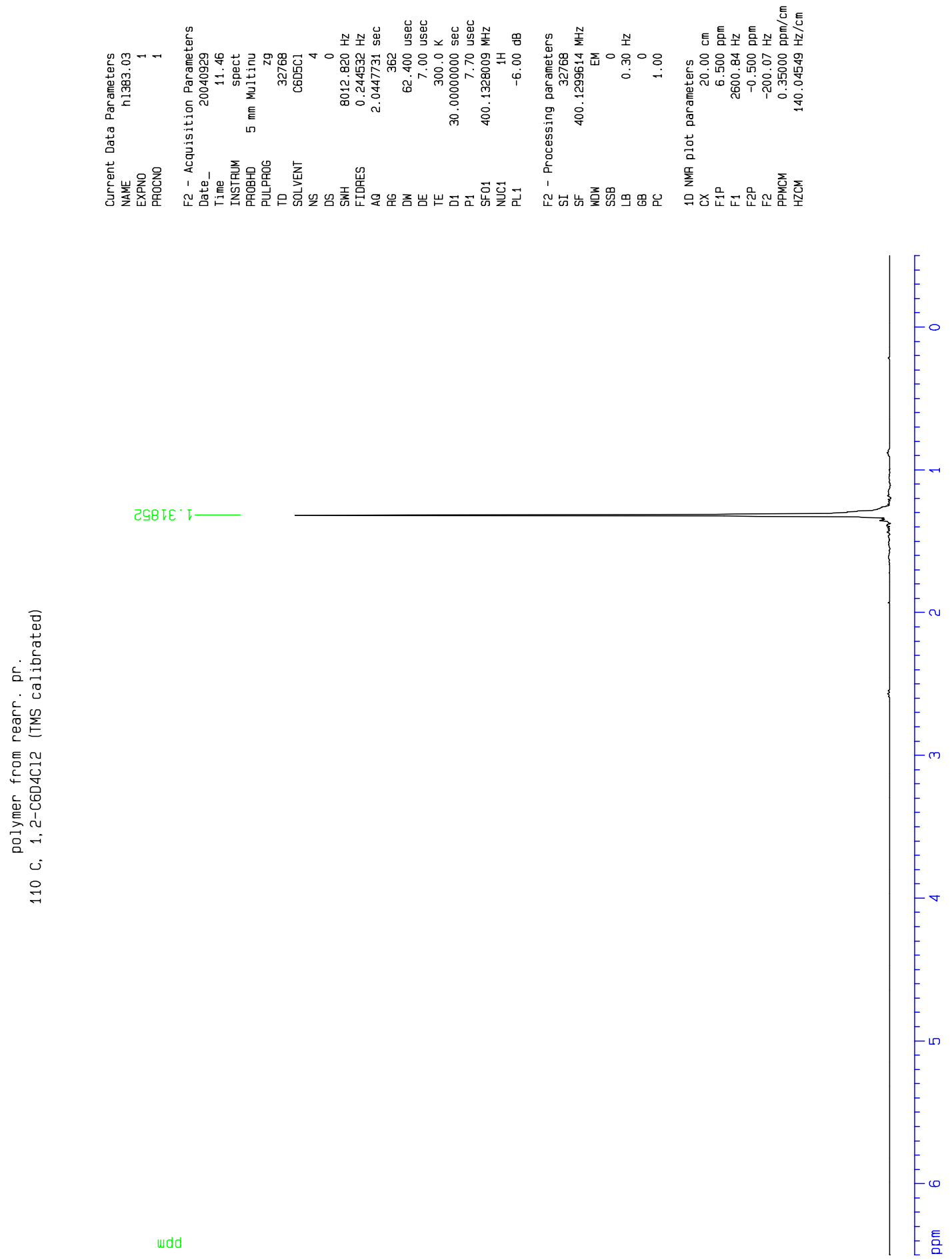

Figure S5. ${ }^{1} \mathrm{H}$ NMR spectrum $\left(110{ }^{\circ} \mathrm{C}\right.$ in $\left.1,2-\mathrm{C}_{6} \mathrm{D}_{4} \mathrm{Cl}_{2}\right)$ of polyethylene produced from 3 at $-78^{\circ} \mathrm{C}$ 

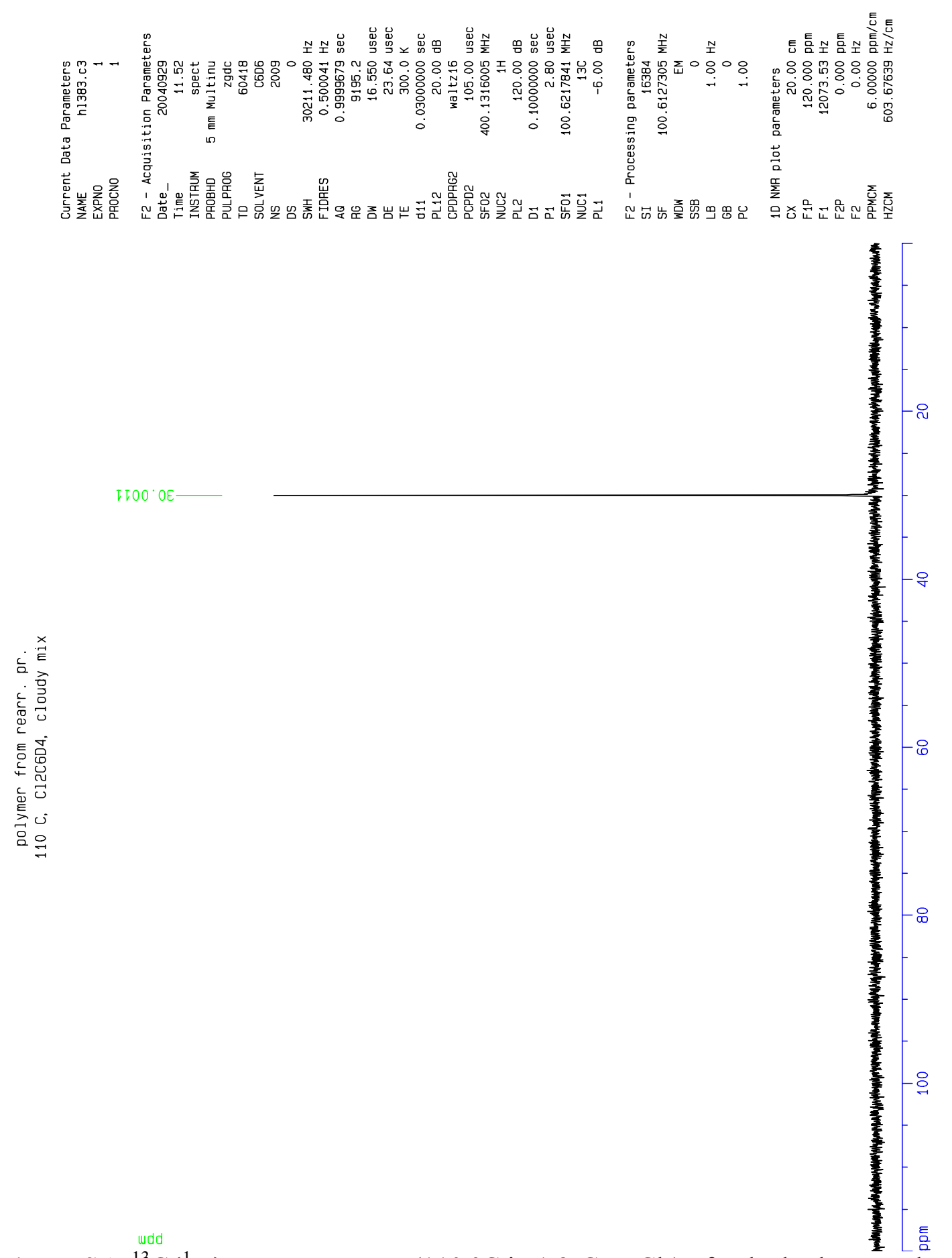

Figure S6. ${ }^{13} \mathrm{C}\left\{{ }^{1} \mathrm{H}\right\}$ NMR spectrum $\left(110{ }^{\circ} \mathrm{C}\right.$ in $\left.1,2-\mathrm{C}_{6} \mathrm{D}_{4} \mathrm{Cl}_{2}\right)$ of polyehtylene produced from 3 at $-78^{\circ} \mathrm{C}$ 


\section{X-Ray Crystallography}

Crystallographic data are summarized in Tables S1. Full details are provided in the accompanying cif files. Data were collected on a Bruker Smart Apex diffractometer using Mo K $\alpha$ radiation $(0.71073 \AA)$. Non-hydrogen atoms were refined with anisotropic displacement coefficients. All hydrogen atoms were included in the structure factor calculation at idealized positions and were allowed to ride on the neighboring atoms with relative isotropic displacement coefficients. The $\mathrm{B}\left(\mathrm{C}_{6} \mathrm{~F}_{5}\right)_{4}{ }^{-}$anion, hydrogen atoms except the $\mu$-BH-Zr, and solvent molecules are omitted from the ORTEP diagrams. All ORTEP diagrams have been drawn with $50 \%$ probability ellipsoids. Specific comments for each structure are as follows.

Tp* $\mathbf{Z r}\left(\mathbf{C H}_{2} \mathbf{P h}\right)_{3}$ (1). Single crystals of $\mathbf{1}$ were obtained by recrystallization from pentane at $-35^{\circ} \mathrm{C}$. The space group was determined as $\mathrm{P} 1$ (bar) based on systematic absences and intensity statistics. Patterson methods were used to locate the $\mathrm{Zr}$ atoms. Repeated difference Fourier maps allowed recognition of all expected C, N and B atoms. Following anisotropic refinement of all non-hydrogen atoms, ideal $\mathrm{H}$ atom positions were calculated. Final refinement was anisotropic for $\mathrm{Zr}, \mathrm{C}, \mathrm{N}$ and B and isotropic-riding for $\mathrm{H}$ atoms. No anomalous bond lengths or thermal parameters were noted.

$\left[\left\{\left(\mathrm{PhCH}_{2}\right)(\mathrm{H}) \mathrm{B}\left(\mu-\mathrm{Me}_{2} \mathrm{pz}\right)_{2}\right\} \mathrm{Zr}\left(\eta^{2}-\mathrm{Me}_{2} \mathrm{pz}\right)\left(\mathrm{CH}_{2} \mathrm{Ph}\right)\right]\left[\mathbf{B}\left(\mathrm{C}_{6} \mathbf{F}_{5}\right)_{4}\right](3)$. Single crystals of 6 were obtained by recrystallization from toluene- $d_{8}$ at $-80{ }^{\circ} \mathrm{C}$. The space group was determined as $\mathrm{P} 2{ }_{1} / \mathrm{c}$ based on systematic absences and intensity statistics. Direct methods were used to locate the $\mathrm{Zr}$ and many $\mathrm{C}$ atoms from the E-map. Repeated difference Fourier maps allowed recognition of all expected C, B and F atoms. Following anisotropic refinement of all non- $\mathrm{H}$ atoms, ideal $\mathrm{H}$ atom positions were 
calculated. Final refinement was anisotropic for $\mathrm{Zr}, \mathrm{C}, \mathrm{F}$ and $\mathrm{B}$ and isotropic-riding for $\mathrm{H}$ atoms. The residual electron density between $\mathrm{Zr}$ and $\mathrm{B}$ was assigned to an $\mathrm{H}$ atom as expected and its position isotropically refined. No anomalous bond lengths or thermal parameters were noted

\section{$\left[\left(\mathrm{CH}_{2} \mathrm{Ph}\right)(\mathrm{H}) \mathrm{B}\left(\mu-\mathrm{Me}_{2} \mathrm{Pz}\right)_{2} \mathrm{Zr}\left(\eta^{2}-\mathrm{Me}_{2} \mathrm{Pz}\right)\left(\mathrm{CH}_{2} \mathrm{Ph}\right)\left(\mathrm{PMe}_{3}\right)\right]\left[\mathrm{B}\left(\mathrm{C}_{6} \mathrm{~F}_{5}\right)_{4}\right] \quad\left(3 \cdot \mathrm{PMe}_{3}\right)$.}

Single crystals of $\mathbf{3} \cdot \mathbf{P M e}_{3}$ were obtained by slow diffusion of hexanes into a concentrated $\mathrm{CH}_{2} \mathrm{Cl}_{2}$ solution. The space group was determined as $\mathrm{P} 2{ }_{1} / \mathrm{c}$ based on systematic absences and intensity statistics. Patterson methods were used to locate the $\mathrm{Zr}$ and some $\mathrm{C}$ atoms. Repeated difference Fourier maps allowed recognition of all expected Zr, C, N, P, F and B atoms. $\mathrm{A} \mathrm{CH}_{2} \mathrm{Cl}_{2}$ molecule was poorly resolved. Following anisotropic refinement of all non- $\mathrm{H}$ atoms, ideal $\mathrm{H}$ atom positions were calculated. Final refinement was anisotropic for all non- $\mathrm{H}$ atoms and isotropic-riding for $\mathrm{H}$ atoms. The residual electron density between $\mathrm{Zr}$ and $\mathrm{B}$ was assigned to an $\mathrm{H}$ atom as expected and its position isotropically refined. The occupancies for the $\mathrm{Cl}$ atoms refined to about 0.96 each. One $\mathrm{C}-\mathrm{Cl}$ distance was short (1.6 angstroms) and the thermal ellipsoid $\mathrm{C}$ atom of the $\mathrm{CH}_{2} \mathrm{Cl}_{2}$ was large and elongated consistent with solvent disorder. No other anomalous bond lengths or thermal parameters were noted.

$\left\{\left(\mathrm{CH}_{2} \mathrm{Ph}\right)(\mathrm{H}) \mathrm{B}\left(\mu-\mathrm{Me}_{2} \mathrm{pz}\right)_{2}\right\} \mathrm{Zr}\left(\eta^{2}-\mathrm{Me}_{2} \mathrm{pz}\right)\left(\eta^{5}-\mathrm{C}_{5} \mathrm{Me}_{5}\right)(\mathrm{Cl})(7)$. Single crystals of 7 were obtained by recrystallization from $\mathrm{CH}_{2} \mathrm{Cl}_{2}$ at $23{ }^{\circ} \mathrm{C}$. The space group was determined as $\mathrm{C} 2 / \mathrm{c}$ based on systematic absences and intensity statistics. Direct methods were used to locate the $\mathrm{Zr}, \mathrm{Cl}$ and most $\mathrm{C}$ atoms from the E-map. Repeated difference Fourier maps allowed recognition of all expected $\mathrm{C}, \mathrm{N}$ and $\mathrm{Cl}$ atoms. Following anisotropic refinement of all non- $\mathrm{H}$ atoms, ideal $\mathrm{H}$ atom positions were calculated. The 
$\mathrm{CH}_{2} \mathrm{Cl}_{2}$ solvent is disordered into two positions and refinement of site occupancy factors were close to 0.5 so were set at 0.5 . Final refinement was anisotropic for all non-H atoms and isotropic-riding for $\mathrm{H}$ atoms. The residual electron density between $\mathrm{Zr}$ and $\mathrm{B}$ was assigned to an $\mathrm{H}$ atom as expected and its position isotropically refined. No anomalous bond lengths or thermal parameters were noted other than slight disorder in the solvent molecule. 
Table S1. Summary of X-Ray Diffraction Data for $\mathbf{1}$ and $\mathbf{3}$

\begin{tabular}{|c|c|c|}
\hline & 1 & 3 \\
\hline formula & $\mathrm{C}_{36} \mathrm{H}_{43} \mathrm{BN}_{6} \mathrm{Zr}$ & $\mathrm{C}_{29} \mathrm{H}_{36} \mathrm{BN}_{6} \mathrm{Zr}+\mathrm{C}_{24} \mathrm{~F}_{20} \mathrm{~B}$ \\
\hline formula weight & 661.79 & 1249.72 \\
\hline crystal system & Triclinic & Monoclinic \\
\hline space group & P1(bar) & $\mathrm{P} 2{ }_{1} / \mathrm{c}$ \\
\hline$a(\AA)$ & $10.654(5)$ & $16.355(6)$ \\
\hline$b(\AA)$ & $12.580(6)$ & $18.565(7)$ \\
\hline$c(\AA)$ & $14.229(7)$ & $16.860(6)$ \\
\hline$\alpha\left({ }^{\circ}\right)$ & $97.674(7)$ & 90.0 \\
\hline$\beta\left(^{\circ}\right)$ & $111.169(7)$ & $97.014(6)$ \\
\hline$\gamma\left({ }^{\circ}\right)$ & $106.607(7)$ & 90.0 \\
\hline$V\left(\AA^{3}\right)$ & $1643.3(14)$ & $5078(3)$ \\
\hline$Z$ & 2 & 4 \\
\hline$T(\mathrm{~K})$ & 100 & 100 \\
\hline crystal color, habit & orange, fragment & yellow, plate \\
\hline GOF on $F^{2}$ & 1.038 & 1.192 \\
\hline $\mathrm{R}$ indices $[I>2 \sigma(I)]^{a}$ & $\mathrm{R} 1=0.0356, \mathrm{wR} 2=0.0816$ & $\mathrm{R} 1=0.0576, \mathrm{wR} 2=0.1166$ \\
\hline $\mathrm{R}$ indices (all data) ${ }^{a}$ & $\mathrm{R} 1=0.0425, \mathrm{wR} 2=0.0839$ & $\mathrm{R} 1=0.0709, \mathrm{wR} 2=0.1224$ \\
\hline
\end{tabular}


Table S2. Summary of X-Ray Diffraction Data for 3·PMe 3 and 7.

\begin{tabular}{|c|c|c|}
\hline & 3. $\mathbf{P M e}_{3}+\mathrm{CH}_{2} \mathrm{Cl}_{2}$ & $7+0.5 \mathrm{CH}_{2} \mathrm{Cl}_{2}$ \\
\hline formula & $\begin{array}{l}\mathrm{C}_{33} \mathrm{H}_{45} \mathrm{BN}_{6} \mathrm{PZr}+\mathrm{C}_{24} \mathrm{~F}_{20} \mathrm{~B}+ \\
\mathrm{CH}_{2} \mathrm{Cl}_{2}\end{array}$ & $\begin{array}{l}\mathrm{C}_{32} \mathrm{H}_{43} \mathrm{BClN}_{6} \mathrm{Zr}+ \\
0.5 \mathrm{CH}_{2} \mathrm{Cl}_{2}\end{array}$ \\
\hline formula weight & 1410.72 (including solvent) & 691.67 (including solvent) \\
\hline crystal system & Monoclinic & Monoclinic \\
\hline space group & $\mathrm{P} 2_{1} / \mathrm{c}$ & $\mathrm{C} 2 / \mathrm{c}$ \\
\hline$a(\AA)$ & $10.793(2)$ & $20.044(4)$ \\
\hline$b(\AA)$ & $28.245(6)$ & $16.076(3)$ \\
\hline$c(\AA)$ & $19.166(4)$ & $20.135(4)$ \\
\hline$\alpha\left({ }^{\circ}\right)$ & 90.0 & 90.0 \\
\hline$\beta\left({ }^{\circ}\right)$ & $92.62(3)$ & $92.33(3)$ \\
\hline$\gamma\left({ }^{\circ}\right)$ & 90.0 & 90.0 \\
\hline$V\left(\AA^{3}\right)$ & $5837(2)$ & $6483(2)$ \\
\hline$Z$ & 4 & 8 \\
\hline$T(\mathrm{~K})$ & 100 & 100 \\
\hline crystal color, habit & orange, fragment & clear, prism \\
\hline GOF on $F^{2}$ & 1.069 & 0.962 \\
\hline $\mathrm{R}$ indices $[I>2 \sigma(I)]^{a}$ & $\mathrm{R} 1=0.0527, \mathrm{wR} 2=0.1144$ & $\mathrm{R} 1=0.0382, \mathrm{wR} 2=0.0766$ \\
\hline $\mathrm{R}$ indices (all data) ${ }^{a}$ & $\mathrm{R} 1=0.0615, \mathrm{wR} 2=0.1191$ & $\mathrm{R} 1=0.0560, \mathrm{wR} 2=0.0810$ \\
\hline
\end{tabular}




\section{ORTEP Diagrams}

Structure of 1

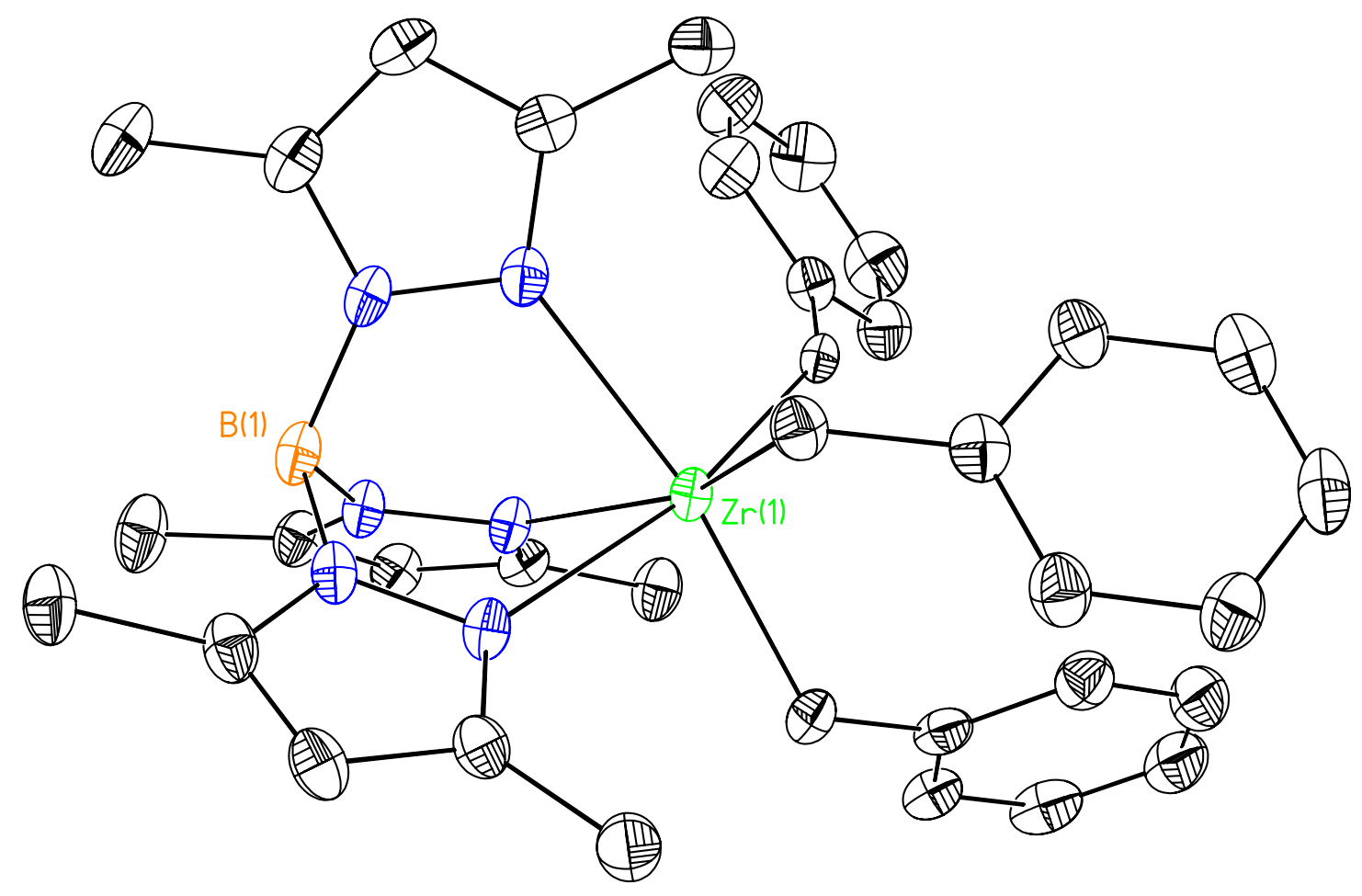


Structure of the cation of $\mathbf{3}$

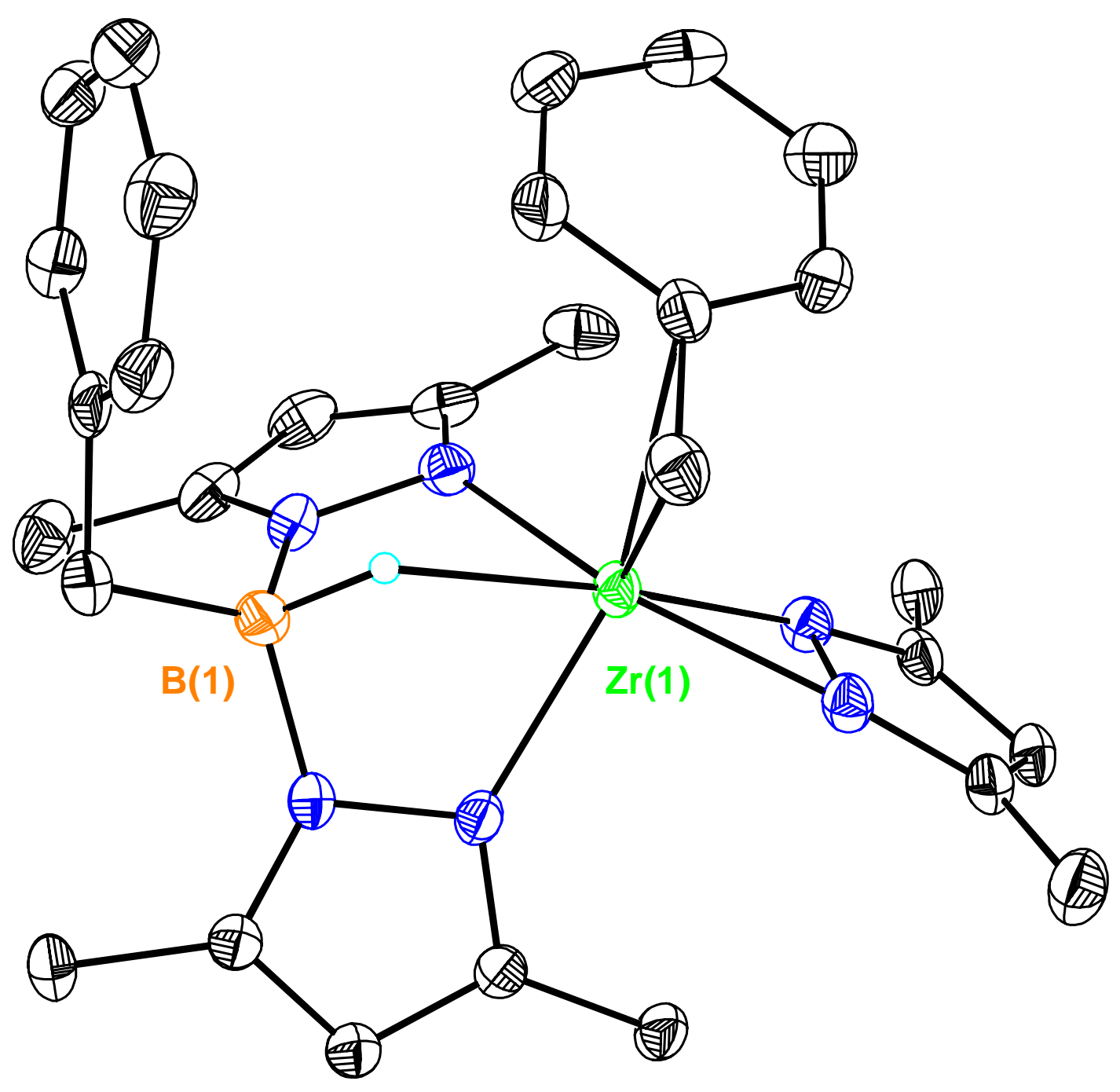


Structure of the cation of $\mathbf{3} \cdot \mathbf{P M e}_{3}$

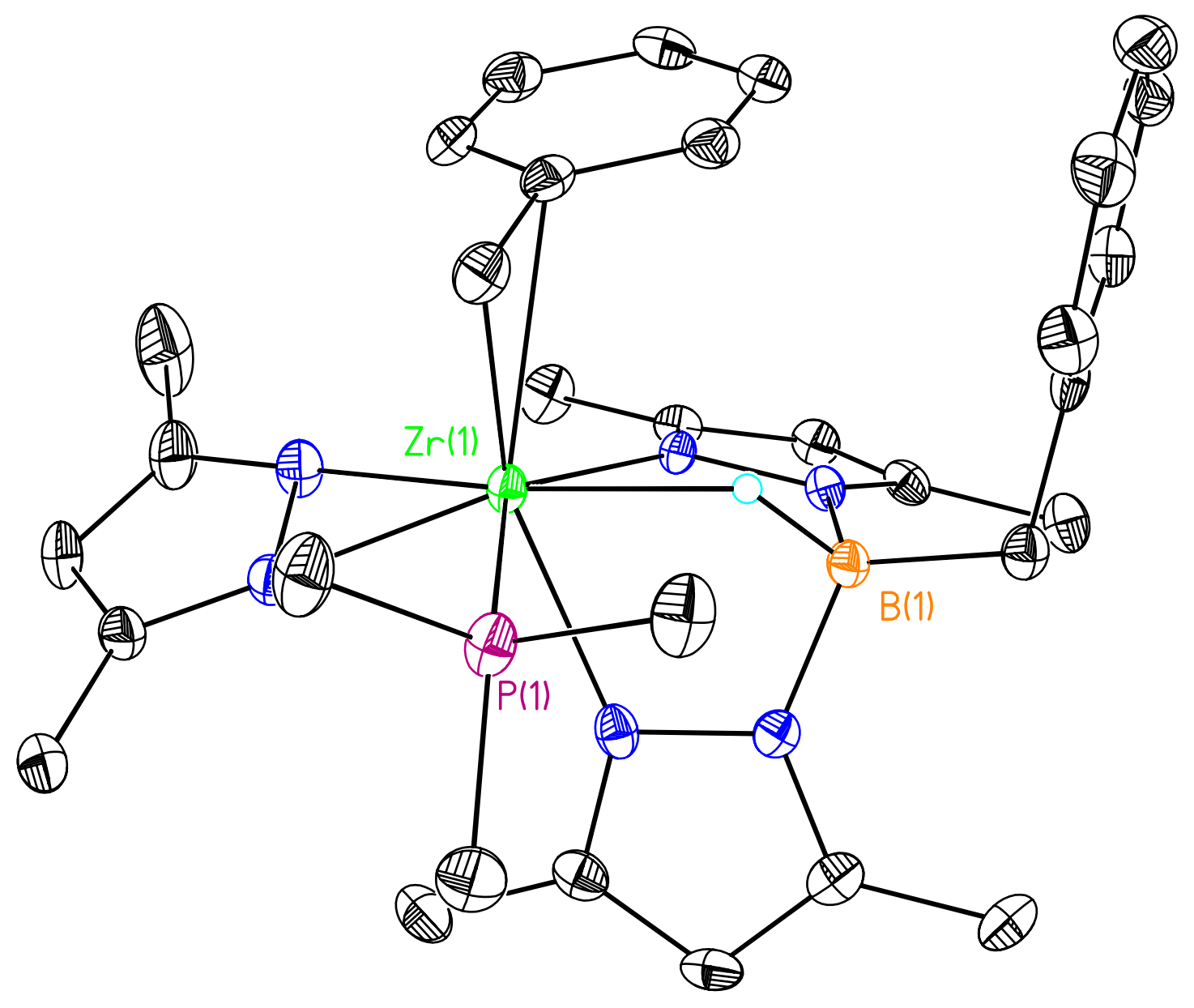


Structure of 7

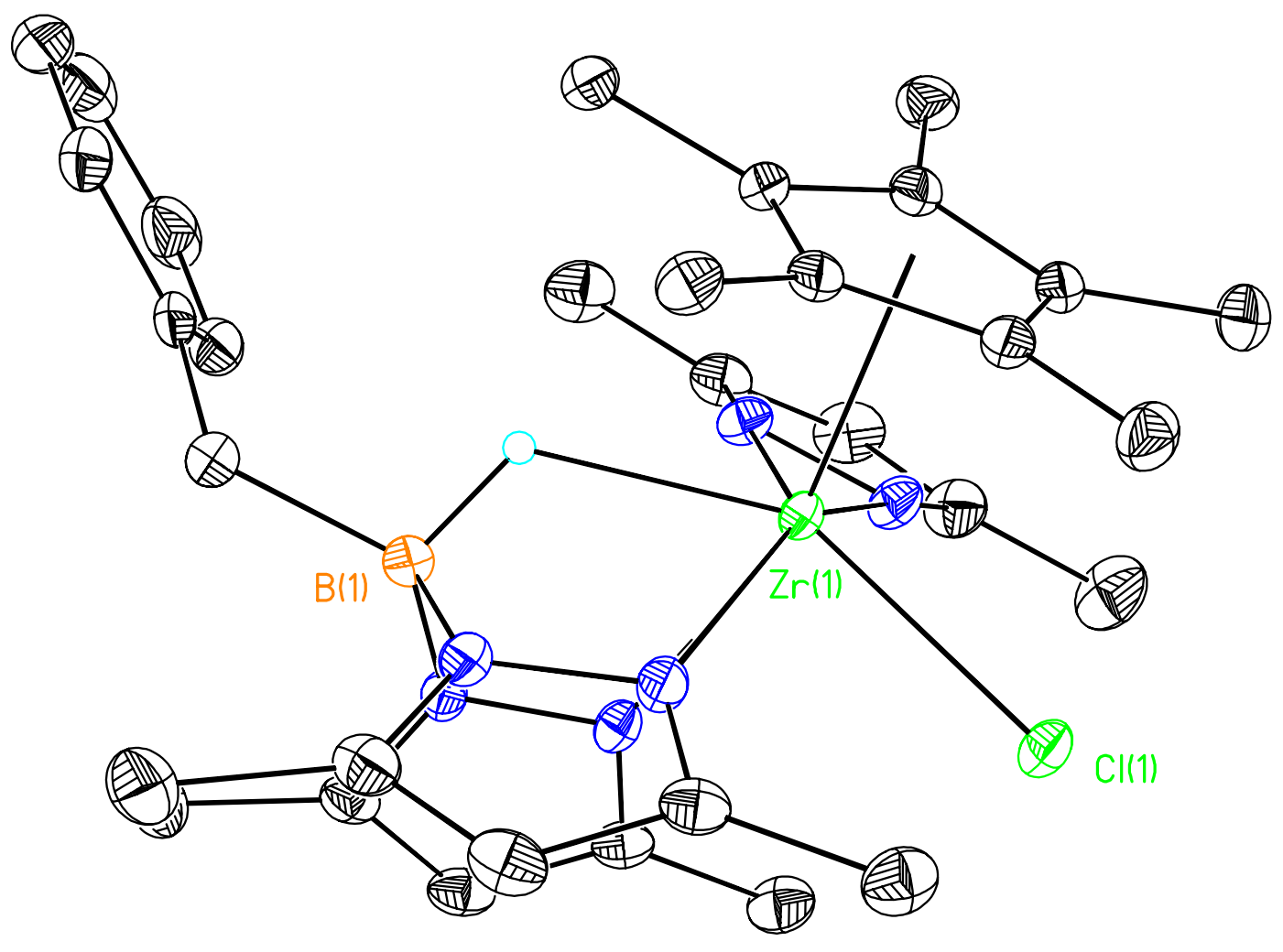

